\title{
Análise da composição química para avaliar o uso do resíduo impureza mineral e vegetal do amendoim na alimentação de bovinos
}

\author{
Submetido - 30 jul. $2020 \quad$ Aprovado - 05 out. $2020 \quad$ Publicado - 14 out. 2020
}

http://dx.doi.org/10.17648/sas.v1i2.54

\begin{abstract}
Aline Schneiders Martins Dalpian $\mathbb{D}$
Mestranda em Administração pela Universidade Estadual Paulista - FCAV/UNESP, Jaboticabal, elaine_sbento@yahoo.com.br.
\end{abstract}

\begin{abstract}
Elaine Bento de Albuquerque (iD)
Mestre em Administração pela Universidade Estadual Paulista - FCAV/UNESP, Jaboticabal, professoraaline48@gmail.com.
\end{abstract}

José de Souza Rodrigues (iD

Professor Assistente da Universidade Estadual Paulista, UNESP/BAURU, jose.rodrigues@unesp.br.

\begin{abstract}
RESUMO
Quanto mais amendoim o Brasil produz, maior também poderá ser a quantidade de resíduo sólido impureza da lavoura. Esse resíduo é originado na lavoura e dentro do contexto de toda a cadeia de produção, passa por várias etapas de separação do amendoim, desde o campo até a indústria beneficiadora. É emergente a necessidade da indústria e dos produtores de amendoim apresentar estratégias mais sustentáveis na cadeia de produção, com a adoção de boas práticas para a contribuição de um planeta mais limpo. Dentre os produtos disponíveis para utilização na nutrição de ruminantes, destacam-se aqueles relacionados com a cultura do amendoim. Apresentar um início de discussão sobre a possibilidade de uso do resíduo impureza mineral e vegetal do amendoim na alimentação de bovinos é o objetivo desse estudo, bem como apresentar o resultado da primeira análise bromatológica para alimentação de bovinos da casca, pele e resíduo da lavoura do amendoim, realizada e divulgada no Brasil.
\end{abstract}

Palavras-chave: Análise Bromatológica; Lavoura; Produção.

\section{Analysis of the chemical composition to evaluate the use of the residue mineral and vegetable impure of peanuts in cattle feeding}

\begin{abstract}
The more peanuts Brazil produces, the greater the amount of solid waste impurity in the crop. This residue originates in the fields and, within the context of the entire production chain, goes through several stages of peanut separation, from the field to the processing industry. The need for peanut industry and producers to present more sustainable strategies in the production chain is emerging, with the adoption of good practices for the contribution of a cleaner planet. Among the products available for use in the nutrition of ruminants, we highlight those related to the cultivation of peanuts. To present a beginning of discussion about the possibility of using the mineral and vegetable impurity residue of peanuts in the feeding of cattle is the objective of this study, as well as to present the result of the first bromatological analysis for feeding cattle of theshell, skin and residue of the crop. peanuts, made and disseminated in Brazil.
\end{abstract}

Keywords: Análisis bromatológico; Agricultura; Producción. 


\title{
Análisis de la composición química para evaluar el uso de residuos minerales y vegetales impuros de maní en alimentación de ganado
}

\begin{abstract}
RESUMEN
Cuantos más cacahuetes produzca Brasil, mayor será la cantidad de impurezas de desechos sólidos en el cultivo. Este residuo se origina en los campos y, en el contexto de toda la cadena de producción, pasa por varias etapas de separación del maní, desde el campo hasta la industria de procesamiento. Se perfila la necesidad de que la industria y los productores de maní presenten estrategias más sostenibles en la cadena productiva, con la adopción de buenas prácticas para el aporte de un planeta más limpio. Entre los productos disponibles para su uso en la nutrición de rumiantes, destacamos los relacionados con el cultivo de maní. Presentar un inicio de discusión sobre la posibilidad de utilizar el residuo de impurezas minerales y vegetales del maní en la alimentación del ganado es el objetivo de este estudio, así como presentar el resultado del primer análisis bromatológico para la alimentación del ganado de la cáscara, piel y residuo del cultivo. maní, elaborado y difundido en Brasil.
\end{abstract}

Palabras clave: Efecto invernadero; Impacto; Sustentabilidad.

\section{Introdução}

O Brasil é o $5^{\circ}$ maior exportador de amendoim do mundo, é fornecedor para a Europa e Emirados Árabes, resultado da qualidade do amendoim produzido no país. Ocupa a $12^{\circ}$ posição no ranking mundial de países produtores de amendoim, e a região de Jaboticabal-SP produz um quarto da tonelada de amendoim exportado, porque o amendoim é cultivado como cultura de rotação com a cana-de-açúcar na região. Esse processo acontece devido sua alta performance na fixação biológica do nitrogênio, melhorando a fertilidade do solo. Para a safra de 2020 no Brasil, a previsão de produção do amendoim é recorde, sendo esperado o equivalente a 516.500 mil toneladas, segundo a CONAB, (2020).

A produção de amendoim gera resíduos de impureza mineral e vegetal como: casca, farelo, grãos chochos e resíduos da lavoura. A casca é destinada para geração de energia, a pele e os grãos chochos são destinados para a produção de óleo e o resíduo impureza mineral e vegetal da lavoura são levados para aterros sanitários.

Os coprodutos são resíduos obtidos a partir de processos da agroindústria (produção de álcool, sucos de fruta, grãos, lavoura e alimentação humana) e, por apresentarem valor nutricional adequado para 
atender a exigência dos animais, podem ser utilizados nas formulações de dietas, substituindo parte do milho e farelo de soja na alimentação animal, permitindo, em alguns casos, o mesmo desempenho a um custo por menor.

No Brasil é cultural o uso de coproduto na alimentação animal, como o caroço de algodão, polpa cítrica, casca de soja e os resíduos de soja. A questão da regionalização é um fator muito importante para a escolha dos coprodutos quando a intenção for reduzir o custo alimentar, pois o processamento, quando necessário e o frete impactam diretamente no preço e a viabilidade da utilização do produto.

Segundo Barbosa (2004), os volumosos são alimentos que possuem mais de $18 \%$ de fibra bruta e menos de $60 \%$ de NDT; já os concentrados seguem na linha contrária, com valores acima de $60 \%$ de NDT, e menos que $18 \%$ de fibra bruta. Dentro dos concentrados, ainda há a classificação em energéticos e proteicos, sendo os energéticos aqueles com menos de $20 \%$ de proteína bruta e os proteicos os ingredientes com mais de $20 \%$ de proteína bruta. Essas informações são úteis para auxiliar o produtor a definir o tipo de coproduto e por qual ingrediente seria possível realizar a substituição.

A pecuária de corte tem expressiva participação no PIB brasileiro, e apresentou uma receita de 433 bilhões em 2017. Segundo o IBGE - Instituto Brasileiro de Geografia e Estatística (2019), apesar de uma queda de 1,5 milhão de cabeças, devido ao aumento dos abates pela indústria de carne, o Brasil segue com o maior rebanho comercial do mundo, com cerca de 213,5 milhões de animais. $O$ valor de movimentações financeiras dado pela cadeia produtiva somou 523,25 bilhões, apresentando aumento de $3,6 \%$, quando comparado ao ano de 2016 e apresenta perspectiva de crescimento nos próximos anos (ABIEC, 2018, 2019).

O Brasil apresenta alta proporção da produção pecuária em condições de pastagens, onde próximo de $87,0 \%$ dos animais abatidos são provenientes da terminação em pastagens, enquanto o restante é terminado em confinamento. Este sistema de produção representa uma vantagem em 
termos de custo de produção, contudo, a estacionalidade da produção e qualidade da forragem proveniente das pastagens resulta em deficiências de nutrientes que comprometem as eficiências agronômica, zootécnica, e ambientais do sistema de produção podem resultar em altos rendimentos. Desta forma, a adoção de técnicas que aumentem a produtividade do rebanho, como o manejo de pastagens, suplementação e terminação em confinamentos garante a alta eficiência dos sistemas (FIGUEIREDO et al., 2007).

De acordo com Leme et al., (2003), a adoção de manejo intensivo, visando maior produtividade exige alto desprendimento de capital, uma vez que grãos e concentrados proteicos possuem alto custo no mercado durante boa parte do ano. Contudo, alternativas que utilizam produtos menos onerosos e de qualidade nutricional na alimentação animal apresentam-se cada vez mais atrativos. Dentre os produtos disponíveis para utilização na nutrição de ruminantes, destacam-se aqueles relacionados com a cultura do amendoim.

De acordo com Heuzé et al. (2017), o fruto (vagem, noz) do amendoim é composto por uma casca externa (21-29\% do peso) e das próprias amêndoas (79-71\% do peso), que consiste das sementes (69-73\%), o germe $(2,0-3,5 \%)$ e a casca fina (testa ou revestimento da semente, correspondendo a $2-3 \%$ do peso), geralmente semelhante a um papel colorido, mais comumente chamado de pele de amendoim.

\section{Material e métodos}

Foi feita uma pesquisa na base científica SCOPUS, com o uso das palavras chave: peanuts in animal feed para encontrar publicações sobre 0 uso dos resíduos casca, farelo, pele e resíduos da lavoura, como alimento ou coproduto na alimentação de ruminantes. Foram considerados somente os últimos 5 anos, tendo sido encontrados 113 títulos e desses, dez foram selecionados. 
Para a determinação da composição química dos coprodutos da cultura do amendoim foram realizadas análises bromatológicas para avaliar os teores de Proteína bruta pelo método de DUMAS (Leco®, modelo FP528, Leco Corporation, Michigan, USA), por meio da combustão a seco, de acordo com os procedimentos da AOAC (1990); Fibra em detergente neutro (FDN) e Fibra em detergente ácido (FDA) determinadas no equipamento Ankom ${ }^{\circledR} 2000$ (Ankom Technologies, New York, USA); Fibra em detergente neutro indigestível (FDNi) - Incubação no rúmen de animais canulados (Método in situ) por 288 horas (VALENTE et al., 2011). O Extrato etéreo foi determinado segundo metodologia proposta por AOCS (2005) e Ankom (2009); O teor de energia bruta (EB) foi determinado em bomba calorimétrica adiabática (PARR Instrument Company 6300, Illinois, USA).

A determinação de FDA foi realizada segundo Robertson e Van Soest (1981), e nitrogênio insolúvel em detergente neutro (NIDN) e nitrogênio insolúvel em detergente ácido (NIDA) conforme recomendações de Licitra et al. (1996).

\section{Resultados e discussão}

O foco da pesquisa realizada foi analisar a composição química no uso do resíduo impureza mineral e vegetal do amendoim na alimentação de bovinos apresentados em estudos de casos publicados em artigos.

O Quadro 1 mostra dez estudos escolhidos com essa temática para discussão, onde são detalhados os anos da pesquisa, os autores, os títulos e os resultados alcançados.

Quadro 1: Resultado de pesquisa na base SCOPUS referentes a pesquisas com subprodutos do amendoim.

\begin{tabular}{|c|l|l|l|}
\hline ANO & \multicolumn{1}{|c|}{ TíTULO } & \multicolumn{1}{c|}{ AUTOR } & \multicolumn{1}{c|}{ RESULTADOS } \\
\hline 2019 & $\begin{array}{l}\text { Neutron activation } \\
\text { analysis and data } \\
\text { mining techniques to } \\
\text { discriminate between } \\
\text { beef cattle diets }\end{array}$ & $\begin{array}{l}\text { Tejeda Mazola, Y., } \\
\text { et al. }\end{array}$ & $\begin{array}{l}\text { Avaliar a composição mineral de } \\
\text { dietas comumente usadas para } \\
\text { alimentar bovinos de corte no } \\
\text { Brasil. }\end{array}$ \\
\hline 2019 & $\begin{array}{l}\text { Inventory and mapping } \\
\text { of food crops waste as } \\
\text { livestock feed }\end{array}$ & $\begin{array}{l}\text { Ali, N., Suhartina, } \\
\text { Muktiani, A., } \\
\text { Pangestu, E. }\end{array}$ & $\begin{array}{l}\text { Inventariar e mapear resíduos } \\
\text { de culturas alimentares como } \\
\text { alimento para o gado para o }\end{array}$ \\
\hline
\end{tabular}




\begin{tabular}{|c|c|c|c|}
\hline & $\begin{array}{l}\text { resources in the } \\
\text { development of beef } \\
\text { cattle in Majene } \\
\text { district, West Sulawesi } \\
\text { Province }\end{array}$ & & $\begin{array}{l}\text { desenvolvimento de bovinos de } \\
\text { corte no distrito de Majene. }\end{array}$ \\
\hline 2019 & $\begin{array}{l}\text { Peanut cake can } \\
\text { replace soybean meal } \\
\text { in supplements for } \\
\text { lactating cows without } \\
\text { affecting production }\end{array}$ & $\begin{array}{l}\text { Dias, C.A.S., } \\
\text { Bagaldo, et al. }\end{array}$ & $\begin{array}{l}\text { Avaliar a substituição total de } \\
\text { farelo de soja por torta de } \\
\text { amendoim no suplemento } \\
\text { concentrado de vacas em } \\
\text { lactação. Amendoim bolo pode } \\
\text { substituir completamente o } \\
\text { farelo de soja na mistura de } \\
\text { concentrado. }\end{array}$ \\
\hline 2018 & $\begin{array}{l}\text { In situ ruminal } \\
\text { degradability of protein } \\
\text { feeds with distinct } \\
\text { physical forms: A } \\
\text { meta-analysis }\end{array}$ & $\begin{array}{l}\text { Busanello, M., } \\
\text { Velho, et al. }\end{array}$ & $\begin{array}{l}\text { Avaliar a degradabilidade } \\
\text { ruminal de alimentos protéicos } \\
\text { com diferentes formas físicas } \\
\text { (pellet versus grão) através de } \\
\text { um estudo de meta-análise. }\end{array}$ \\
\hline 2017 & $\begin{array}{l}\text { Intake, digestibility, } \\
\text { performance, and } \\
\text { nitrogen metabolism of } \\
\text { feedlot-finished young } \\
\text { bulls (Bos indicus) fed } \\
\text { diets containing } \\
\text { peanut cake }\end{array}$ & Correia, B.R., et al. & $\begin{array}{l}\text { Avaliar o uso de torta de } \\
\text { amendoim como substituto } \\
\text { dietético do farelo de soja e } \\
\text { determinar os efeitos no } \\
\text { consumo, digestibilidade } \\
\text { aparente, desempenho e } \\
\text { metabolismo do } \mathrm{N} \text { em touro } \\
\text { jovens terminados em } \\
\text { confinamento. }\end{array}$ \\
\hline 2016 & $\begin{array}{l}\text { Meat quality of lambs } \\
\text { fed diets with peanut } \\
\text { cake }\end{array}$ & Bezerra, L.S et al. & $\begin{array}{l}\text { Substituição do farelo de soja } \\
\text { pela torta de amendoim foi } \\
\text { avaliada na qualidade da carne } \\
\text { de } 45 \text { cordeiros mestiços Dorper } \\
\text { x Santa Inês. }\end{array}$ \\
\hline 2016 & $\begin{array}{l}\text { Carcass traits and } \\
\text { meat quality of } \\
\text { crossbred boer goats } \\
\text { fed peanut cake as a } \\
\text { substitute for soybean } \\
\text { meal }\end{array}$ & $\begin{array}{l}\text { Silva, T.M., de } \\
\text { Medeiros, et al. }\end{array}$ & $\begin{array}{l}\text { Determinar o impacto da } \\
\text { substituição do farelo de soja } \\
\text { pela torta de amendoim nas } \\
\text { dietas de cabras Boer mestiças. }\end{array}$ \\
\hline 2016 & $\begin{array}{l}\text { Intake and digestibility, } \\
\text { rumen fermentation, } \\
\text { and concentrations of } \\
\text { metabolites in steers } \\
\text { fed with peanut cake }\end{array}$ & $\begin{array}{l}\text { de Oliveira, P.A., } \\
\text { Oliveira, et al. }\end{array}$ & $\begin{array}{l}\text { Avaliar a torta de amendoim } \\
\text { como substituto do farelo de } \\
\text { soja na dieta de novilhos. }\end{array}$ \\
\hline 2016 & $\begin{array}{l}\text { Nutritional values and } \\
\text { antioxidative activities } \\
\text { of whole peanuts and } \\
\text { peanut skins for } \\
\text { ruminant feeds }\end{array}$ & $\begin{array}{l}\text { Saito, C., Asano, et } \\
\text { al. }\end{array}$ & $\begin{array}{l}\text { Investigar os valores nutricionais } \\
\text { de dois subprodutos do } \\
\text { amendoim, planta inteira do } \\
\text { amendoim não padronizados e } \\
\text { peles de amendoim, juntamente } \\
\text { com seus efeitos no } \\
\text { crescimento e fermentação } \\
\text { microbiana no rúmen, seus } \\
\text { valores nutritivos e suas } \\
\text { atividades antioxidantes em um } \\
\text { teste de digestão usando quatro } \\
\text { cabras. }\end{array}$ \\
\hline
\end{tabular}




\begin{tabular}{|l|l|l|l|}
\hline 2016 & $\begin{array}{l}\text { Production and quality } \\
\text { of beef from young } \\
\text { bulls fed diets } \\
\text { supplemented with } \\
\text { peanut cake }\end{array}$ & Correia, B.R., et al. & $\begin{array}{l}\text { Avaliar a importância da } \\
\text { inclusão da torta de amendoim } \\
\text { na dieta sobre a taxa de } \\
\text { crescimento de touros jovens, } \\
\text { produção e qualidade da carne. }\end{array}$ \\
\hline
\end{tabular}

Fonte: Elaborado pelos autores com dados da base SCOPUS (2020).

Nota-se que foram realizados mais experimentos com a torta de amendoim como coproduto para alimentação animal, e os resultados, na sua maioria, foram positivos quanto ao uso dessa biomassa. Segundo Saito et al. (2016), amendoim inteiro e amendoim em peles podem ser usados nas dietas de alta energia e alta proteína para ruminantes, e as peles de amendoim pode ser considerada um alimento com funções antioxidantes.

Dias et al. (2018) avaliaram a substituição total de farelo de soja por torta de amendoim no suplemento concentrado de vacas em lactação e identificaram que a torta de amendoim pode substituir completamente o farelo de soja na mistura de concentrado, porque não altera a ingestão da matéria seca, a produção e composição de leite, e em adição ele pode reduzir o custo da alimentação.

Em estudo conduzido por Duarte et al. (2015), foi determinado os efeitos de níveis de torta de amendoim em substituição ao farelo de soja (0; 33,33 ; 66,67 ; e $100 \%$ ) no suplemento concentrado de cordeiros. Os consumos de matéria seca, proteína bruta, nutrientes digestíveis totais, fibra em detergente neutro e fibra em detergente ácido, bem como coeficientes de digestibilidade da matéria seca, fibra em detergente neutro e carboidratos não fibrosos, não foram afetados pela inclusão da torta de amendoim.

Pode-se observar experimentos com resultados diferentes utilizando o mesmo animal, touros jovens, por exemplo. O experimento que utilizou touros jovens (sem identificar a raça), apresentou resultado favorável ao uso do bolo do amendoim como coproduto na alimentação do animal. Já para o touro jovem de uma outra raça específica não foi favorável a substituição de coproduto de amendoim. Os resultados demonstraram que são necessárias 
mais pesquisas para comparação de resultados entre animais de mesma raça, bem como também de raças diferentes.

Utley et al. (1973) avaliaram as dietas de acabamento com alto conteúdo de energia, contendo $72 \%$ de milho moído, $8 \%$ de suplemento proteico e $20 \%$ de cascas de amendoim não moídas, moídas e peletizadas como fontes de volumoso e observaram que a ingestão, os ganhos médios diários e as características da carcaça foram semelhantes nos novilhos alimentados com dietas contendo as três formas de processamento da casca de amendoim.

No entanto, foram observadas variações nos ganhos de peso corporal e uma alta incidência (56 a 60\%) de abscesso hepático nos lotes de novilhos alimentados com casca de amendoim moída ou peletizada. Por outro lado, os novilhos alimentados com cascas não moídas responderam com ganhos de peso corporal relativamente uniforme e apenas um dos 27 novilhos teve abscessos no fígado.

Este estudo indica que a alteração da forma física das cascas de amendoim não moídas para uma forma moída ou peletizada reduz seu valor como fibra efetiva e demonstra a necessidade de usar cascas não moídas quando usadas como fonte de volumosos.

\section{Análise Bromatológica para alimentação de bovinos}

A avaliação da composição químico-bromatológica das amostras de amendoim pele, amendoim casca e amendoim resíduo foi realizada no laboratório da Faculdade de Ciência Agrária e Veterinária da UNESP, localizada na cidade de Jaboticabal-SP, com o intuito de verificar os teores dos nutrientes presentes para avaliar o possível uso desses coprodutos na alimentação de bovinos.

A Tabela 1 apresenta os resultados da análise bromatológica feita com a casca, pele e resíduos da lavoura do amendoim. 
Tabela 1: Resultados da análise bromatológica dos coprodutos de amendoim.

\begin{tabular}{|c|c|c|c|c|c|}
\hline Amostra & $1^{\text {a }}$ MS (\%) & 2a MS (\%) & MS (\%) & MM (\%MS) & MO1 \% \\
\hline Amendoim Pele & 96,40 & 94,62 & 91,21 & 2,70 & 97,30 \\
\hline $\begin{array}{c}\text { Amendoim Casca } \\
\text { Amendoim }\end{array}$ & 94,54 & 93,26 & 88,16 & 4,48 & 95,52 \\
\hline Resíduo & 93,16 & 94,55 & 88,08 & 13,69 & 86,31 \\
\hline${ }^{1} \mathrm{MO}$ : Matéria orgânic & & & & & \\
\hline Amostra & \multicolumn{2}{|c|}{ PB (\%MS) } & \%PIDN (PB) & \multicolumn{2}{|c|}{ \%PIDA (PB) } \\
\hline Amendoim Pele & \multicolumn{2}{|c|}{23,74} & 42,23 & \multicolumn{2}{|c|}{16,43} \\
\hline Amendoim Casca & \multicolumn{2}{|c|}{13,26} & 39,82 & \multicolumn{2}{|c|}{33,76} \\
\hline Amendoim Resíduo & \multicolumn{2}{|c|}{11,96} & 34,70 & \multicolumn{2}{|c|}{29,01} \\
\hline
\end{tabular}

\begin{tabular}{|c|c|c|c|c|c|c|c|c|}
\hline \multicolumn{2}{|c|}{ Amostra } & \multicolumn{2}{|c|}{ EE (\%MS) } & \multicolumn{3}{|c|}{ Energia (cal/g) } & \multicolumn{2}{|c|}{ Energia $(\mathrm{MJ} / \mathrm{Kg})$} \\
\hline Amendoi & n Pele & & 7,94 & & 5731 & & 23,9 & \\
\hline Amendoin & Casca & & 6,03 & & 4739 & & 19,8 & \\
\hline Amendoim & Resíduo & & 4,52 & & 4179 & & 17,5 & \\
\hline Amostra & $\begin{array}{c}\text { FDN } \\
(\% M S)\end{array}$ & $\begin{array}{c}\text { FDA } \\
\text { (\%MS) }\end{array}$ & $\begin{array}{c}\text { FDNi } \\
(\% M S)\end{array}$ & $\begin{array}{c}\text { FDNcp }{ }^{1} \\
(\%)\end{array}$ & $\begin{array}{c}\text { CIDN } \\
\text { (\%MS) }\end{array}$ & $\begin{array}{c}\text { CIDA } \\
\text { (\%MS) }\end{array}$ & $\begin{array}{c}\text { PIDN } \\
\text { (\%MS) }\end{array}$ & $\begin{array}{c}\text { PIDA } \\
\text { (\%MS) }\end{array}$ \\
\hline $\begin{array}{l}\text { Amendoim } \\
\text { Pele } \\
\text { Amendoim }\end{array}$ & 49,08 & 43,46 & 31,30 & 37,83 & 1,22 & 0,47 & 10,03 & 3,90 \\
\hline $\begin{array}{c}\text { Casca } \\
\text { Amendoim }\end{array}$ & 62,78 & 56,98 & 50,98 & 55,75 & 1,75 & 1,03 & 5,28 & 4,48 \\
\hline Resíduo & 54,59 & 48,71 & 42,83 & 47,10 & 3,35 & 2,61 & 4,15 & 3,47 \\
\hline
\end{tabular}

O resíduo amendoim apresentou alto teor de matéria mineral $(\mathrm{MM})$, 13,69\%, reduzindo, assim, o teor de matéria orgânica presente no coproduto. O teor mais elevado de matéria mineral em relação aos outros coprodutos avaliados pode ser devido à forma de obtenção do resíduo, agregando contaminantes como a terra.

Dessa forma, sugere-se que para a utilização desse coproduto deve ser avaliada uma forma de limpeza do resíduo com o intuito de reduzir a contaminação e avaliar os possíveis contaminantes presentes na amostra, a fim de não proporcionar riscos à saúde dos animais, pois alguns minerais em altos teores podem ser tóxicos ao animal. Além disso, com o aumento dos processos necessários a avaliação para a possível utilização desse coproduto, os custos agregados podem inviabilizar economicamente a sua utilização.

O coproduto amendoim pele apresentou teor de proteína bruta superior a $20 \%$, podendo ser caracterizado como um alimento proteico, 
enquanto os coprodutos amendoim resíduo e amendoim casca apresentaram teores de proteína bruta (PB) de 10,47 e 11,61\%. No entanto, a fração da proteína bruta ligada à fibra insolúvel em detergente ácido (PIDA) foi superior no amendoim casca. A fração PIDA é considerada indisponível para utilização, dessa forma, deve ser considerada na inclusão desse coproduto no fornecimento de proteína bruta.

Por meio das análises de PIDN (Proteína Insolúvel em Detergente Neutro) e PIDA (Proteína Insolúvel em Detergente Ácido) foi possível determinar a fração B3 da proteína bruta, ou seja, a fração de proteína verdadeira de baixa degradação ruminal, pois está ligada à parede celular. Esse valor foi superior no amendoim pele, reduzindo assim, as frações de proteína prontamente disponíveis e as frações de maior degradação ruminal.

Ainda, o amendoim pele apresentou maior teor de extrato etéreo (27,94\%), sendo necessário avaliar o nível de inclusão nas dietas para ruminantes em função do alto extrato etéreo.

Os teores de fibra insolúvel em detergente neutro (FDN) e fibra insolúvel em detergente neutro indigestível (FDNi) dos coprodutos foram avaliados, com maior teor encontrado no amendoim casca $62,78 \%$ e $50,98 \%$, respectivamente. Para todos os coprodutos avaliados, os teores de FDNi foram altos, principalmente para o amendoim casca, devendo ser avaliada a qualidade da fibra e o intuito da inclusão dessa fibra na dieta dos ruminantes para não causar enchimento ruminal, podendo reduzir 0 consumo e a digestibilidade dos nutrientes.

Portanto, as análises bromatológicas realizadas apresentam resultados que contribuem para continuação de pesquisas referentes a inclusão da pele, casca e resíduo do amendoim como coproduto na alimentação animal, porém são necessários experimentos e novas análises para avaliação. Nas pesquisas futuras, orienta-se determinar os níveis de inclusão de cada coproduto, para identificar o nível máximo de inclusão visando as características de cada um. 
Apesar de haver experimentos com o uso do amendoim em grão, e seus resíduos como o bolo, a casca, a farinha, e a pele do amendoim, não foi possível encontrar estudos com o uso do resíduo impureza mineral e vegetal da lavoura na alimentação de bovinos. A análise bromatológica nos apresenta uma possível causa da não utilização do resíduo impureza mineral e vegetal da lavoura na alimentação animal, que é a quantidade de mineral encontrado nesse resíduo.

\section{Conclusões}

Portanto, os resíduos da produção do amendoim que apresentam potencial para uso na alimentação de bovinos, devido as suas características proteicas e de fibras são: a casca e a pele. $O$ resíduo impureza da lavoura, na sua forma original, apresenta considerável quantidade de mineral, e seu uso para a alimentação de bovinos é inapropriado. A análise bromatológica apresenta resultado favorável para o uso da pele e da casca do amendoim na alimentação de bovinos, e mostra uma possível causa da não utilização do resíduo impureza mineral e vegetal da lavoura, devido a quantidade de mineral encontrado na sua composição. Sugere-se novos estudos com a possibilidade de separação da impureza mineral, e aproveitamento dos arbustos, grãos e cascas que compõem esse resíduo. O uso deste resíduo para alimentação animal, ou outros usos, evitaria a destinação dele para aterros sanitários como ocorre atualmente no Brasil, aumentando o impacto ambiental da cultura do amendoim. Como trabalhos futuros é necessário analisar as condições sanitárias a serem observadas para o uso do resíduo para alimentar animais.

\section{Agradecimentos}

Agradecemos ao Professor Dr. Ricardo Andrade Reis docente da área de Forragicultura e Pastagens da Faculdade de Ciências Agrárias e Veterinárias, Câmpus de Jaboticabal e a Ronyatta Weich Teobaldo, doutoranda em Zootecnia também nesse câmpus, pela contribuição com os resultados obtidos na análise bromatológica. 


\section{Referências}

ALI, N. SUHARTINA, MUKTIANI, A. PANGESTU, E. Inventory and mapping of food crops waste as livestock feed resources in the development of beef cattle in Majene district, West Sulawesi Province. International Journal of Scientific and Technology Research 8(9), pp. 28-31

ANKOM. Technology method 2: rapid determination of oil/fat utilizing high temperature solvent extraction. Macedon, p. 2. 2009.

AOAC - Association of Official Agricultural Chemists. Official Methods of Analysis of the Association of Official Analytical Chemists. Washington, DC: Association of Official Analytical Chemists, 771 p. 1990.

AOCS. American Oil Chemists' Society. Official Method Am 5-04, Rapid determination of oil/fat utilizing high temperature solvent extraction. Urbana: Official Methods and Recommended Pratices of the American Oil Chemists' Socity, 2005.

BARBOSA, F.A. Alimentação na nutrição de bovinos. Produção Animal da Escola de Veterinária/UFMG - junho/2004. Disponível em: http://www.agronomia

.com.br/conteudo/artigos/artigos_nutricao_bovinos.htm. Acesso em: $25 / 07 / 2020$

BEZERRA, L.S. et al. Meat quality of lambs fed diets with peanut cake. Meat Science 121, pp. 88-95.

BUSANELLO, M. VELHO, et al. In situ ruminal degradability of protein feeds with distinct physical forms: A meta-analysis. Emirates Journal of Food and Agriculture 30(3), pp. 240-244.

CONAB - Companhia Nacional De Abastecimento. Safra Brasileira de Grãos: Boletim Grãos Maio 2020 Completo. Disponível em: https://www.conab. gov.br/info-agro/safras/graos. Acesso em: 23/07/2020.

CORREIA, B.R. et al. Intake, digestibility, performance, and nitrogen metabolism of feedlot-finished young bulls (Bos indicus) fed diets containing peanut cake. Journal of Animal Science 94(11), pp. 4720-4727.

Production and quality of beef from young bulls fed diets supplemented with peanut cake. Meat Science 118, pp. 157-163

DIAS, C.A.S. BAGALDO, et al. Peanut cake can replace soybean meal in supplements for lactating cows without affecting production. Tropical Animal Health and Production 50(3), pp. 651-657.

FIGUEIREDO, D. M. et al. Análise econômica de quatro estratégias de suplementação para recria e engorda de bovinos em sistema pastosuplemento. Revista Brasileira de Zootecnia, v. 36, n. 5, p. 1443-1453, 2007. 
DUARTE, R. A. B. et al. Torta de amendoim em substituição ao farelo de soja na alimentação de cordeiros $1 / 2$ sangue Dorper. Arch. Zootec., v. $64, n$. 248, p. 317-322, 2015.

LICITRA, G. HERNANDEZ, T.M. VAN SOEST, P.J. (1996) Standardization of procedures for nitrogen fractionation of ruminant feeds. Animal Science Feed Technology 57: 347.1996.

MAZOLA, Y.T. et al. Neutron activation analysis and data mining techniques to discriminate between beef cattle diets. Journal of Radioanalytical and Nuclear Chemistry 322(3), pp. 1571-1578.

OLIVEIRA, P.A. OLIVEIRA, et al. Intake and digestibility, rumen fermentation, and concentrations of metabolites in steers fed with peanut cake. Tropical Animal Health and Production 48(2), pp. 403-409.

ROBERTSON, J.B. VAN SOEST P.J.V. The detergent system of analysis. In: JAMES, W. P. T., THEANDER, O. (Eds.). The Analysis of Dietary Fibre in Food. Marcel Dekker, NY, Chapter 9, p. 123-158. 1981.

SAITO, C. ASANO, S. et al. Nutritional values and antioxidative activities of whole peanuts and peanut skins for ruminant feeds. Animal Science Journal 87 (1), pp. 54-60

SILVA, T.M. DE MEDEIROS A.N. et al. Carcass traits and meat quality of crossbred boer goats fed peanut cake as a substitute for soybean meal. Journal of Animal Science 94 (7), pp. 2992-3002

UTLEY, P. R. MCCORMICK, W. C. Level of Peanut Hulls as a Roughage Source in Beef Cattle Finishing Diets. Journal of Animal Science, 34 (1):146-151. 1972.

VALENTE T.N.P. DETMANN E. QUEIROZ A.C. VALADARES FILHO S.C. GOMES D.I. FIGUEIRAS J.F. Evaluation of ruminal degradation profiles of forages using bags made from different textiles. Revista Brasileira de Zootecnia 40:2565-2573. 2011. 